\title{
Three-Dimensional Drawings of Bounded Degree Trees ${ }^{\star}$
}

\author{
Fabrizio Frati and Giuseppe Di Battista \\ Università di Roma Tre \\ \{frati,gdb\}@dia.uniroma3.it
}

\begin{abstract}
We show an algorithm for constructing $3 D$ straight-line drawings of balanced constant degree trees. The drawings have linear volume and optimal aspect ratio. As a side effect, we also give an algorithm for constructing $2 D$ drawings of balanced constant degree trees in linear area, with optimal aspect ratio and with better angular resolution with respect to the one of [8]. Further, we present an algorithm for constructing $3 D$ poly-line drawings of trees whose degree is bounded by $n^{1 / 3}$ in linear volume and with optimal aspect ratio.
\end{abstract}

\section{Introduction}

The problem of constructing $3 D$ drawings of trees with limited volume is interesting both in practice and in theory and it has attracted the attention of several researchers. Since a $2 D$ drawing is also a $3 D$ drawing then the results known for two-dimensional drawings of trees are still valid in $3 D$. However, embedding a $2 D$ drawing in three dimensions fills the space only in one of its planes, while one would prefer a drawing uniformally distributed in the embedding space. A widely used measure for expressing this is given by the aspect ratio of a drawing, that is the ratio between the maximum and the minimum edge of its bounding box. Clearly, considering a $2 D$ drawing of an $n$-nodes tree as a $3 D$ drawing yields a bad $\left(O\left(n^{1 / 2}\right)\right)$ aspect ratio.

The state of the art in $2 D$ can be summarized as follows. No algorithm is known for drawing an $n$-nodes tree in $O(n)$ area and such a bound is achieved only in special cases. For example, if the degree of the nodes is bounded by $n^{1 / 2}$, then the algorithm of Garg and Rusu [7] constructs $O(n)$ area straight-line drawings. As another example, complete trees can be drawn straight-line in linear area with the algorithm of Trevisan [8]. Concerning algorithms that work in three dimensions, Felsner et al. [5] have shown how to draw in $3 D$ any outerplanar graph and so any tree using linear volume. The drawings constructed by such an algorithm have bad $(O(n))$ aspect ratio. In fact, they lie on the surface of a $O(n)$ length triangular prism. However, the problem of finding linear volume $3 D$ drawings of trees with good aspect ratio is still open.

In this paper we contribute to the above problems: (1) In Section 3 we show how to adapt the algorithm in [3] for constructing a linear volume $3 D$ drawing of a balanced tree with degree bounded by a constant. The aspect ratio is $O(1)$. (2) As a side effect of our technique we give an algorithm for drawing in $2 D$ a balanced tree whose degree is bounded by a constant in linear area, with constant aspect ratio and $\Omega(1 / \sqrt{n})$ angular resolution (Section 4). This improves the results of Trevisan that in [8] showed an

\footnotetext{
* Work partially supported by "Project ALGO-NEXT: Algorithms for the Next Generation Internet and Web: Methodologies, Design, and Experiments", MIUR PRIN.
} 
algorithm for constructing drawings with the same area and aspect ratio, but with only $O(1 / n)$ angular resolution. (3) In Section 5, we show how to construct a poly-line $3 D$ drawing of a tree with degree bounded by $n^{1 / 3}$ in $O(n)$ volume and $O(1)$ aspect ratio.

\section{Preliminaries}

We assume familiarity with trees and their drawings [4] and assume that trees are rooted. The degree of a node is the number of its children. The degree of a tree is the maximum degree of one of its nodes. The height of a tree is the maximum length (number of nodes) of a path from the root to a leaf. In the following we call $T_{h}$ a complete tree with height $h$. We call $r_{h}$ its root and, if the degree of $T_{h}$ is $k, T_{1, h-1}, T_{2, h-1}, \ldots T_{k, h-1}$ the subtrees of $T_{h}$ rooted at the children of $r_{h}$. We call such children $r_{1, h-1}, r_{2, h-1}, \ldots r_{k, h-1}$. For complete trees the number of nodes is a function of $h$ and $k$. Namely, $n=1+k+$ $k^{2}+\ldots+k^{h-1}=\frac{k^{h}-1}{k-1}$. Hence $k^{h}=n(k-1)+1$ and so $h=\log _{k}[n(k-1)+1]$. A balanced tree is such that its height is logarithmic in the number of its nodes.

Grid drawings, straight-line drawings, and poly-line drawings are defined as usually ([4]). The bounding box $B(\Gamma)$ of a drawing $\Gamma$ is the smallest rectangle $(2 D)$ or parallelepiped (3D) with edges parallel to the coordinate axes, that covers $\Gamma$ completely. We denote by left $(B(\Gamma))$, right $(B(\Gamma))$, back $(B(\Gamma)), \operatorname{front}(B(\Gamma)), \operatorname{bot}(B(\Gamma))$ and top $(B(\Gamma))$ the sides of $B(\Gamma)$. In the $2 D$ case $x$ grows from left to right and $y$ from bottom to top. In the $3 D$ case $x$ grows from left to right, $y$ from back to front and $z$ from bottom to top. The aspect ratio of $\Gamma$ is the ratio between the maximum and the minimum edge of $B(\Gamma) . \Gamma$ is (strictly) upward in one coordinate direction if, for each node, such coordinate is (less than) not greater than the same coordinate of its children. The angular resolution of $\Gamma$ is the minimum angle between two segments incident to the same node. $\Gamma$ satisfies the subtree separation property ([1]) if, for any two node-disjoint subtrees of $T$, the bounding boxes of their partial drawings don't intersect. $\Gamma$ satisfies the tip-over property $([\overline{8}])$ if, for any node, its children are drawn on a line parallel to one coordinate axis. In the following we call $x$-line, $y$-line or $z$ line a line parallel to the $x$-axis, $y$-axis or $z$-axis, respectively. Analogously, we call $x y$-plane, $x z$-plane or $y z$-plane a plane parallel to the coordinate planes $x y, x z$ and $y z$, respectively.

\section{Three-Dimensional Straight-Line Drawings of Balanced Constant Degree Trees}

In the following we show an algorithm to draw a balanced constant degree tree $T$ in three dimensions. First, add extra nodes to $T$ until it is complete. This can be done without altering the height $h$ and the degree $k$ of $T$. Now we have to construct a drawing $\Gamma_{h}$ of a complete tree $T_{h}$. This can be done recursively as follows. If $h=1$, then place $r_{1}$ in $(0,0,0)$. If $h>1$, suppose you have drawn $\Gamma_{1, h-1}, \Gamma_{2, h-1}, \ldots, \Gamma_{k, h-1}$. We distinguish three cases: (i) if $h \bmod 3 \equiv 2$, then place $\Gamma_{1, h-1}, \Gamma_{2, h-1}, \ldots, \Gamma_{k, h-1}$ so that left $\left(\Gamma_{1, h-1}\right), \ldots, \operatorname{left}\left(\Gamma_{k, h-1}\right)$ are on the same $y z$-plane, so that $\operatorname{back}\left(\Gamma_{1, h-1}\right), \ldots$, $\operatorname{back}\left(\Gamma_{k, h-1}\right)$ are on the same $x z$-plane and so that $\operatorname{top}\left(\Gamma_{i, h-1}\right)$ is one unit below 
$\operatorname{bot}\left(\Gamma_{i+1, h-1}\right), \forall i$ such that $1 \leq i<k$. Place $r_{h}$ one unit to the left and on the same $x$ line of $r_{1, h-1}$ (see Fig.1(a)); (ii) if $h \bmod 3 \equiv 0$, then place $\Gamma_{1, h-1}, \Gamma_{2, h-1}, \ldots, \Gamma_{k, h-1}$ so that $\operatorname{bot}\left(\Gamma_{1, h-1}\right), \ldots, \operatorname{bot}\left(\Gamma_{k, h-1}\right)$ are on the same $x y$ plane, so that $\operatorname{back}\left(\Gamma_{1, h-1}\right)$, $\ldots, \operatorname{back}\left(\Gamma_{k, h-1}\right)$ are on the same $x z$-plane and so that $\operatorname{right}\left(\Gamma_{i, h-1}\right)$ is one unit to the left of left $\left(\Gamma_{i+1, h-1}\right), \forall i$ such that $1 \leq i<k$. Place $r_{h}$ one unit behind and on the same $y$ line of $r_{1, h-1}$ (see Fig. 1 (b)); (iii) if $h \bmod 3 \equiv 1$, then place $\Gamma_{1, h-1}, \Gamma_{2, h-1}, \ldots, \Gamma_{k, h-1}$ so that $\operatorname{bot}\left(\Gamma_{1, h-1}\right), \ldots, \operatorname{bot}\left(\Gamma_{k, h-1}\right)$ are on the same $x y$ plane, so that left $\left(\Gamma_{1, h-1}\right), \ldots, \operatorname{le} f t\left(\Gamma_{k, h-1}\right)$ are on the same $y z$-plane and so that $\operatorname{front}\left(\Gamma_{i, h-1}\right)$ is one unit behind $\operatorname{back}\left(\Gamma_{i+1, h-1}\right), \forall i$ such that $1 \leq i<k$. Place $r_{h}$ one unit below and on the same $z$ line of $r_{1, h-1}$ (see Fig. 1 (c)). Finally, remove from $T_{h}$ the extra nodes and their incident edges to obtain a drawing $\Gamma$ of $T$. The algorithm we have just described is the main ingredient in the proof of the following theorem.

Theorem 1. Given an $n$-nodes balanced tree $T$ with height $h$ and constant degree $k$, there exists an $O(n)$ time algorithm that constructs a $3 D$ crossing free straight-line grid drawing $\Gamma$ of $G$ such that: the volume is $O(n)$, the aspect ratio is $O(1), \Gamma$ satisfies the subtree separation property, $\Gamma$ satisfies the tip-over property, and $\Gamma$ is (strictly) upward in each of the three coordinate directions.

Proof (sketch): We construct a straight-line drawing $\Gamma$ of $T$ by applying the algorithm described in this section. By inductive arguments it's easy to show that $\Gamma$ is crossingfree and satisfies the subtree separation property and the tip-over property. Further, by an easy inductive analysis, it is possible to prove that $\Gamma_{h}$ (and so $\Gamma$ ) is contained in a bounding box $B\left(\Gamma_{h}\right)$ of dimension $[O(\sqrt[3]{n}) \times O(\sqrt[3]{n}) \times O(\sqrt[3]{n})],[O(\sqrt[3]{n / k}) \times$ $\left.O(\sqrt[3]{n / k}) \times O\left(\sqrt[3]{n k^{2}}\right)\right]$, or $\left[O(\sqrt[3]{n k}) \times O\left(\sqrt[3]{n / k^{2}}\right) \times O(\sqrt[3]{n k})\right]$ if $h \bmod 3 \equiv 1$, if $h \bmod 3 \equiv 2$, or if $h \bmod 3 \equiv 0$, respectively. Since $k=O(1)$ the bounds on the volume and on the aspect ratio of $\Gamma$ follow. It's easy to see that $\Gamma$ is upward in each of the three coordinate directions. A slight modification of the algorithm permits also to produce strictly upward drawings: for this purpose, it is sufficient to translate, in the inductive construction of the algorithm, the drawings of the subtrees $T_{1, h-1}, T_{2, h-1}, \ldots$, $T_{k, h-1}$ by vectors $(1,0,1),(1,1,0)$ and $(0,1,1)$, for the case in which $h \bmod 3 \equiv 0$, $h \bmod 3 \equiv 1$ and $h \bmod 3 \equiv 2$, respectively. Such a modification doesn't alter the asymptotic bounds on the volume and on the aspect ratio of $\Gamma$. Finally, the algorithm can be easily implemented to run in linear time.

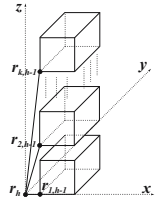

(a)

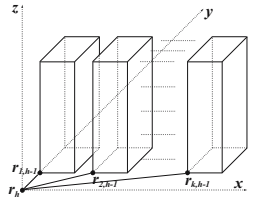

(b)

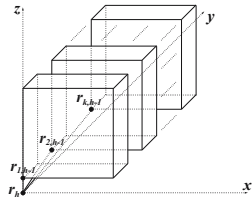

(c)

Fig. 1. Inductive construction of $\Gamma_{h}$ : (a) $h \bmod 3 \equiv 2$. (b) $h \bmod 3 \equiv 0$. (c) $h \bmod 3 \equiv 1$. 


\section{Two-Dimensional Drawings of Constant Degree Balanced Trees}

We now apply a variation of the algorithm in Section 3 to draw a balanced constant degree tree $T$ in two dimensions. First, add extra nodes to $T$ until it is complete. Again, this can be done without altering the height $h$ and the degree $k$ of $T$. Now we have to construct a drawing $\Gamma_{h}$ of a complete tree $T_{h}$. This can be done recursively as follows. If $h=1$, then place $r_{1}$ in $(0,0)$. If $h>1$, suppose you have drawn $\Gamma_{1, h-1}, \Gamma_{2, h-1}, \ldots, \Gamma_{k, h-1}$. We distinguish two cases: (i) if $h$ is even, then place $\Gamma_{1, h-1}, \Gamma_{2, h-1}, \ldots, \Gamma_{k, h-1}$ so that $\operatorname{bot}\left(\Gamma_{1, h-1}\right), \ldots, \operatorname{bot}\left(\Gamma_{k, h-1}\right)$ are on the same $x$ line and so that $\operatorname{le} f t\left(\Gamma_{i+1, h-1}\right)$ is one unit to the right of $\operatorname{right}\left(\Gamma_{i, h-1}\right), \forall i$ such that $1 \leq i<k$. Place $r_{h}$ one unit below and on the same $y$-line of $r_{1, h-1}$ (see Fig. 2(a)); (ii) if $h$ is $o d d$, then place $\Gamma_{1, h-1}, \Gamma_{2, h-1}, \ldots, \Gamma_{k, h-1}$ so that le $f t\left(\Gamma_{1, h-1}\right), \ldots$, le ft $\left(\Gamma_{k, h-1}\right)$ are on the same $y$-line and so that $\operatorname{bot}\left(\Gamma_{i+1, h-1}\right)$ is one unit above $\operatorname{top}\left(\Gamma_{i, h-1}\right)$, $\forall i$ such that $1 \leq i<k$. Place $r_{h}$ one unit to the left and on the same $x$-line of $r_{1, h-1}$ (see Fig. 2(b)). Finally, remove from $T_{h}$ the extra nodes and their incident edges to obtain a drawing $\Gamma$ of $T$. We have the following theorem:

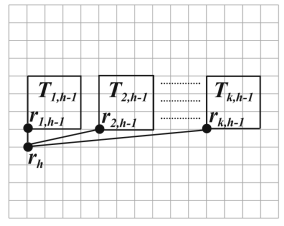

(a)

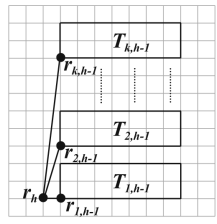

(b)

Fig. 2. Inductive construction of $\Gamma_{h}$ : (a) $h$ even. (b) $h$ odd.

Theorem 2. Given an n-nodes balanced tree $T$ with height $h$ and constant degree $k$, there exists an $O(n)$ time algorithm that constructs a $2 D$ planar straight-line grid drawing $\Gamma$ of $T$ such that: the area is $O(n)$, the aspect ratio is $O(1)$, the angular resolution is $\Omega(1 / \sqrt{n}), \Gamma$ satisfies the tip-over property, $\Gamma$ satisfies the subtree separation property, and $\Gamma$ is (strictly) upward in each of the two coordinate directions.

Proof (sketch): We construct a straight-line drawing $\Gamma$ of $T$ by applying the algorithm described in this section. By inductive arguments it's easy to show that $\Gamma$ is planar and satisfies the subtree separation property and the tip-over property. Further, by an easy inductive analysis, it is possible to prove that $\Gamma_{h}$ (and so $\Gamma$ ) is contained in a bounding box $B\left(\Gamma_{h}\right)$ of dimension $[O(\sqrt{n}) \times O(\sqrt{n})]$, or $[O(\sqrt{n k}) \times O(\sqrt{n / k})]$, if $h$ is odd, or if $h$ is even, respectively. Since $k=O(1)$ the bounds on the area and on the aspect ratio of $\Gamma$ follow. It's easy to see that $\Gamma$ is upward in each of the three coordinate directions. A slight modification of the algorithm similar to that described in Section 3 permits also to produce strictly upward drawings without altering the asymptotic bounds on the area and on the aspect ratio of $\Gamma$. We now analyze the angular resolution of $\Gamma$. It is possible to show by induction that the angle between segments $\overline{r_{k-1, h-1} r_{1, h}}$ and $\overline{r_{k, h-1} r_{1, h}}$, say $\phi$, is the smallest angle in $\Gamma_{h}$. We call $l$ the length of the longest edge of $B\left(\Gamma_{h}\right)$. So $l$ is the number of grid points on the longest edge of $B\left(\Gamma_{h}\right)$ minus one, and so 


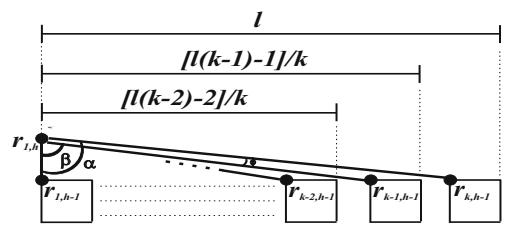

Fig. 3. Angular resolution of $\Gamma_{h}$

$l=O(\sqrt{n k})$. We now derive the value of $\sin (\phi)$ by applying the trigonometric formula $\sin (\phi)=\sin (\alpha) \cos (\beta)-\sin (\beta) \cos (\alpha)$ to the angles $\alpha, \beta$, and $\phi$ shown in Fig. 3 and by applying the Pythagorean Theorem to the two rectangular triangles between vertices $r_{1, h}, r_{1, h-1}, r_{k-1, h-1}$, and $r_{k, h-1}$ :

$$
\begin{aligned}
\sin (\phi) & =\frac{\left(\frac{k-1}{k} l-\frac{1}{k}+1\right)-\left(\frac{k-2}{k} l-\frac{2}{k}+1\right)}{\sqrt{\left(\frac{k-1}{k} l-\frac{1}{k}+1\right)^{2}+1} \sqrt{\left(\frac{k-2}{k} l-\frac{2}{k}+1\right)^{2}+1}}>\frac{\frac{l+1}{k}}{(l+1)^{2}+1}> \\
> & \frac{l}{k\left(l^{2}+2 l+2\right)}=\Omega\left(\frac{1}{k l}\right)=\Omega\left(\frac{1}{k^{\frac{3}{2}} \sqrt{n}}\right)=\Omega\left(\frac{1}{\sqrt{n}}\right) .
\end{aligned}
$$

Finally, the algorithm can be easily implemented to run in linear time.

The table below compares some asymptotic properties of the algorithm shown in this section with those of the algorithm of Trevisan ([8]).

\begin{tabular}{ccccc}
\hline algorithm & area & aspect ratio & angular resolution & subtree separation \\
\hline Our Algorithm & $O(n)$ & $O(1)$ & $\Omega(1 / \sqrt{n})$ & YES \\
\hline Algorithm $[8]$ & $O(n)$ & $O(1)$ & $O(1 / n)$ & NO \\
\hline
\end{tabular}

\section{Three-Dimensional Poly-line Drawings of Bounded Degree Trees}

This section is devoted to the proof of the following theorem:

Theorem 3. Given a n-nodes tree $T$ with degree $k=O\left(n^{\delta}\right)$, where $\delta$ is a constant less than $\frac{1}{3}$, there exists a three-dimensional poly-line crossing-free drawing $\Gamma$ with $O(n)$ volume and $O(1)$ aspect ratio.

The proof of the above theorem strongly exploits the techniques introduced in [6] by Garg et al. They showed that given two constants $\delta$ and $\alpha$, with $0<\delta<\alpha<1$, for every $n$-nodes tree $T$ with degree $k=O\left(n^{\delta}\right)$ it is possible to construct a twodimensional upward planar poly-line grid drawing $\Gamma^{\prime}$ with $O(n)$ area, height $H=$ $O\left(n^{1-\alpha}\right)$ and width $W=O\left(n^{\alpha}\right)$. This is done as follows: (1) $T$ is augmented with dummy nodes to an homeomorphic tree $T^{\prime}$; (2) each node $v$ of $T^{\prime}$ is associated with a layer $\gamma(v)$, so that for each edge $(u, v)$ of $T^{\prime}|\gamma(u)-\gamma(v)| \leq 1$; (3) it is constructed a planar straight-line drawing of $T^{\prime}$ with the property that $y(v)=\gamma(v)$ for each vertex $v$; (4) each dummy node is replaced by a bend, obtaining the poly-line drawing $\Gamma^{\prime}$ of $T$. 
To obtain a three-dimensional drawing $\Gamma$ of $T$ with the properties claimed in Theorem 3, we suppose to apply the algorithm in [6]. Now we perform a "roll up" of $\Gamma^{\prime}$, in a way very similar to that used in [2] to transform two-dimensional orthogonal drawings in three-dimensional drawings. This is done as follows. First, subdivide $\Gamma^{\prime}$ in $O\left(H^{1 / 2}\right)$ drawings $\Gamma_{0}^{\prime}, \Gamma_{1}^{\prime}, \ldots, \Gamma_{k}^{\prime}$, so that $\Gamma_{i}^{\prime}$ contains the part of $\Gamma^{\prime}$ between layers $i \cdot\left\lfloor H^{1 / 2}\right\rfloor$ and $(i+1) \cdot\left\lfloor H^{1 / 2}\right\rfloor-1$ (see Fig. $\left.4(\mathrm{~b})\right)$. So the height of each $\Gamma_{i}^{\prime}$ is $O\left(H^{1 / 2}\right)$. Then we move each $\Gamma_{i}^{\prime}$ to the plane $z=i$ and we reflect each $\Gamma_{i}^{\prime}$ such that $i$ is odd with respect to $x y$-plane (see Fig. 4 (c)). More precisely, the transformation of $\Gamma^{\prime}$ in $\Gamma$ consists in assigning the three coordinates to each vertex and to each bend so that: (1) the $x$-coordinate of each vertex (bend) $v$ of $T$ is equal to the $x$-coordinate of $v$ in $\Gamma^{\prime}$; (2) denoting by $y^{*}(v)$ the $y$-coordinate of $v$ in $\Gamma^{\prime}$, the $y$-coordinate of each vertex (bend) $v$ of $T$ that belongs to $\Gamma_{i}^{\prime}$, with $i$ even (odd), is set equal to $y^{*}(v)-i \cdot\left\lfloor H^{1 / 2}\right\rfloor$ (resp. equal to $\left.(i+1) \cdot\left\lfloor H^{1 / 2}\right\rfloor-y^{*}(v)-1\right)$; (3) the $z$-coordinate of each vertex (bend) $v$ of $T$ that belongs to $\Gamma_{i}^{\prime}$ is equal to $i$. From [6], we know that by setting $\alpha$ to $1 / 3, \Gamma^{\prime}$ has height $H=O\left(n^{2 / 3}\right)$ and width $W=O\left(n^{1 / 3}\right)$. Further, by our construction, the $y$-extension of $\Gamma$ is $H^{1 / 2}=O\left(n^{1 / 3}\right)$ and the $z$-extension of $\Gamma$ is equal to the number of drawings $\Gamma_{i}^{\prime}$, i.e. $O\left(n^{1 / 3}\right)$. So the volume and aspect ratio bounds claimed in Theorem 3 follow. From the planarity of $\Gamma^{\prime}$ and from the property that each segment of such drawing belongs to one layer or is between two consecutive layers it is easy to derive that $\Gamma$ is crossing-free.

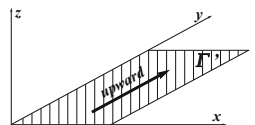

(a)

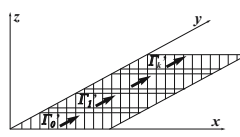

(b)

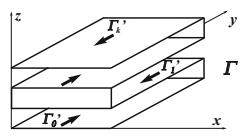

(c)

Fig. 4. (a) A planar poly-line upward grid drawing $\Gamma^{\prime}$ of $T$. (b) Subdivision of $\Gamma^{\prime}$ in partial drawings $\Gamma_{0}^{\prime}, \Gamma_{1}^{\prime}, \ldots, \Gamma_{k}^{\prime}$,. (c) Roll up of $\Gamma^{\prime}$ in a three-dimensional drawing $\Gamma$.

\section{References}

1. T. M. Chan, M. T. Goodrich, S. Rao Kosaraju, and R. Tamassia. Optimizing area and aspect ratio in straight-line orthogonal tree drawings. Comput. Geom., 23(2):153-162, 2002.

2. R. F. Cohen, P. Eades, T. Lin, and F. Ruskey. Three-dimensional graph drawing. Algorithmica, 17(2):199-208, 1997.

3. P. Crescenzi, G. Di Battista, and A. Piperno. A note on optimal area algorithms for upward drawings of binary trees. Comput. Geom., 2:187-200, 1992.

4. G. Di Battista, P. Eades, R. Tamassia, and I. G. Tollis. Graph Drawing. Prentice Hall, Upper Saddle River, NJ, 1999.

5. S. Felsner, G. Liotta, and S. K. Wismath. Straight-line drawings on restricted integer grids in two and three dimensions. J. Graph Algorithms Appl., 7(4):363-398, 2003.

6. A. Garg, M. T. Goodrich, and R. Tamassia. Planar upward tree drawings with optimal area. Int. J. Comput. Geometry Appl., 6(3):333-356, 1996.

7. A. Garg and A. Rusu. Straight-line drawings of general trees with linear area and arbitrary aspect ratio. In ICCSA (3), pages 876-885, 2003.

8. L. Trevisan. A note on minimum-area upward drawing of complete and Fibonacci trees. Information Processing Letters, 57(5):231-236, 1996. 